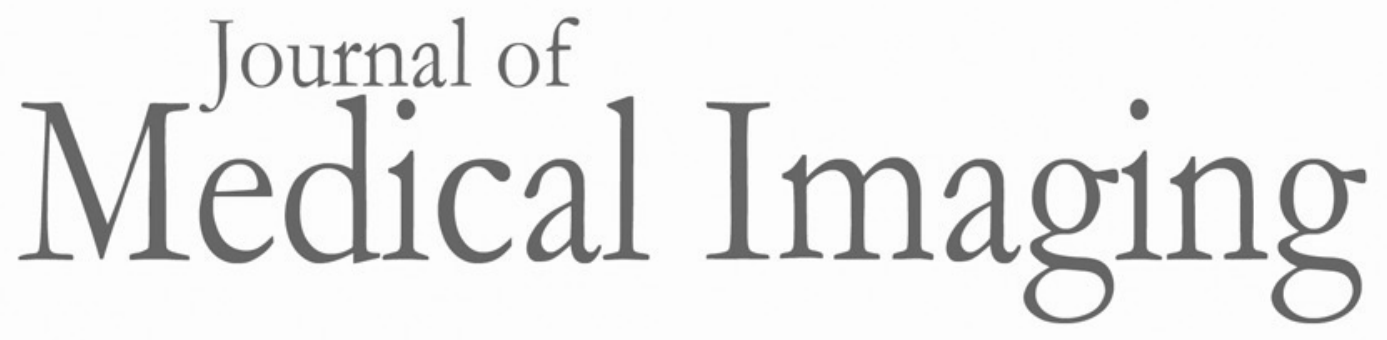

Medicallmaging.SPIEDigitalLibrary.org

\title{
Technology Platforms for Treatment and Discovery in Human Systems: Novel Work in Image-Guided Procedures, Robotic Interventions, and Modeling
}

\author{
Amber Simpson \\ Michael Miga
}




\title{
Technology Platforms for Treatment and Discovery in Human Systems: Novel Work in Image-Guided Procedures, Robotic Interventions, and Modeling
}

\author{
Amber Simpson \\ Memorial Sloan Kettering Cancer Center \\ Department of Surgery \\ New York, New York, United States \\ E-mail: simpsonl@mskcc.org \\ Michael Miga \\ Vanderbilt University \\ Department of Biomedical Engineering \\ Nashville, Tennessee, United States \\ E-mail: michael.i.miga@ vanderbilt.edu
}

This special section on image-guided procedures, robotic interventions, and modeling for the Journal of Medical Imaging is primarily concerned with applications of medical imaging data in the engineering of therapeutic systems. The call for papers for the special section was released in spring 2017, and we received a total of 30 submissions. Each of the papers was sent by the guest editors to one of the guest associate editors who oversaw rigorous peer review. This special section was extremely well subscribed, and the papers accepted comprise one of the largest JMI special sections in the four-year history of the journal.

The accepted papers represent an exceptionally innovative cross section of approaches aimed at improving surgical, interventional, and diagnostic care of patients. Exemplifying the patient-centered nature of the special section, $39 \%$ of articles report on human data in real-world clinical scenarios (Bonmati et al.; Gerard et al.; Heiselman et al.; Jin et al.; Rathore et al.; Xia et al.; Xia et al.; Tian et al; Zhang et al.). Notably, several contributions highlight timely work in women's health issues. Not surprisingly, several articles reflect the heightened interest in machine learning. Other contributions move the needle on therapeutic systems with novel system design. Below, we describe five themes identified in the collective body of work.

\section{Applications in women's health}

Three papers describe applications that address health concerns specific to women, an often underrepresented group in medical imaging research. These applications include teleoperated hysterectomy (Allard, Shubert, and Bell), pelvic floor disorder assessment (Bonmati et al.), and twin-to-twin transfusion syndrome (Tella-Amo et al.).

\section{Integration of machine learning}

Four papers reflect recent intensified interest in machine learning (deep learning) and data science in medical imaging, applied here in the context of therapeutic systems. Acknowledging the need for large datasets in deep learning, Zheng et al. trained a convolutional neural network (CNN) with a combination of synthetic and real images for $2 \mathrm{D}$ to

(C) 2018 Society of Photo-Optical Instrumentation Engineers (SPIE)
$3 \mathrm{D}$ registration. Tian et al. propose a CNN-based prostate segmentation algorithm evaluated with $140 \mathrm{MRI}$ scans. Bonmati et al. supplemented training data with multiple images of the same patient for fully automated ultrasound examination. Reflecting the increased interest in radiomics (the extraction of high dimensional information from medical images), Rathore et al. used features extracted from multiparametric MRI to predict recurrence in glioblastoma patients undergoing radiotherapy.

\section{Real-world systems}

Several papers detail the use of novel systems with an emphasis on clinical workflow and real-world use of technology. A variety of clinical applications are described, representing the growing need for application-specific solutions. Andress et al. describe a portable surgical support system capable of augmented reality in any operating room, which aims to minimize the disruptions in surgical workflow inherent in many surgical guidance systems. Xia and Breen propose an automated image registration method to optimally position patients for radiotherapy to improve image quality and registration accuracy. Edgcumbe et al. provide an end-to-end projector-based system for laparoscopic partial nephrectomy in which the real-time position of the tumor is provided during resection. Zhang et al. describe a system to calculate the overlap of neural stimulation in cochlear implants including a comparison with previously described work. Linte et al. modeled and visualized lesions for real-time navigation inside the beating heart for cardiac ablative therapy, with the goal of reducing the re-operation rate for this complex procedure. Fotouhi et al. propose intraoperative component planning which incorporates two C-arm images for the correct alignment of the implant in hip replacement surgery. Nguyen et al. explored the overlay of fluorescence imaging on tissue for surgical guidance. The integration of molecular imaging into surgical workflows has the potential to augment surgical displays with cancer detection capabilities.

Lastly, there are two novel studies aimed at augmenting image-guided surgery systems with computational models to correct for misalignment due to deformations of soft tissue. Gerard et al. propose a novel approach combining intraoperative ultrasound, augmented reality, and patient-specific models for correcting brain deformation in image-guided 
neurosurgery systems. Heiselman et al. provide a detailed study describing liver deformation during minimally invasive laparoscopic surgery followed by a complete realization of a novel approach to image-to-physical registration using patient-specific models and sparse laparoscopic surface data.

\section{Robotic systems and sensing}

Novel combinations of robotics and sensors are proposed for a variety of clinical applications. Cheng et al. propose photoacoustic-based catheter tracking where a piezoelectric sensor is placed at the tip of the catheter. The pose of the photoacoustic markers is determined by a stereo-camera. Yi et al. describe a robotically held drill guide for supplanting traditional surgical navigation. Zhang et al. discuss a needle insertion platform where a single ultrasound transducer is mounted to the tip of a needle, and sweeping the needle forms images. Lastly, the work by Uff et al. demonstrate the impact of tumor mobility on axial strain elastograms used within the context of soft tissue discrimination when detecting lesions.

\section{Mock surgery validation and software tools}

Novel phantoms were proposed in two papers: Laing et al. describe a patient-specific cardiac phantom for training and surgical planning, and Jin et al. propose a dynamic phantom with breathing motion. Brown et al. provide 3D printing tools for constructing optical tracking targets. In the work by Xia et al., robust techniques are demonstrated in ultrasound segmentation for measuring geometry and pathology of mitral valves for the purpose of recapitulating these characteristics in a patient-specific phantom.

The papers in this special section represent a complex scaffolding of technologies, all primarily focused on one goal - to treat disease with human-scale systems. From the perspective of clinical outcomes, this is, of course, a laudable and important goal. However, we also note that these systems provide an incredible opportunity to study other important aspects of disease in humans. In fact, many of these investigations, while addressing a fundamental clinical need, are a first step towards developing instrumentation to facilitate correlative studies between therapy and disease. For example, the Heiselman et al. study focuses on understanding the nature of deformation during laparoscopic liver procedures and proposes a method to re-establish precise tumor localization; this tool would greatly facilitate investigations into disease recurrence within the context of locoregional therapies. It is nearly impossible to optimize therapies when one is uncertain of the relationship between the target and all other forms of image-based information.

Scanning the special section topics, we see example after example of investigations focused on the precise relationships among the intraoperative tissue domain, available patient-specific preoperative and intraoperative sensing, and the delivery of a therapy. These investigations represent the power of image guidance to impact the study of human disease and to inform quantitative measurement of outcomes in response to therapeutics.
We thank the following guest associate editors for their dedication and contribution to the special section:

Wolfgang Birkfellner, Medizinische Univ. Wien (Austria)

Brett Byram, Vanderbilt Univ. (United States)

Elvis C. S. Chen, Robarts Research Institute (Canada)

Sandrine de Ribaupierre, Western Univ. (Canada)

Baowei Fei, Emory Univ. (United States)

George J. Grevera, Saint Joseph's Univ. (United States)

David Hawkes, Univ. College London (United Kingdom)

David R. Holmes, Mayo Clinic (United States)

Pierre Jannin, Univ. de Rennes 1 (France)

David M. Kwartowitz, Grand Canyon Univ. (United States)

Cristian A. Linte, Rochester Institute of Technology (United States)

Lena Maier-Hein, Deutsches Krebsforschungszentrum (Germany)

Parvin Mousavi, Queen's Univ. (Canada)

Jack H. Noble, Vanderbilt Univ. (United States)

Maryam E. Rettmann, Mayo Clinic (United States)

Eric J. Seibel, Univ. of Washington (United States)

Alexander Seitel, Deutsches Krebsforschungszentrum (Germany)

Guy Shechter, Philips Healthcare (United States)

Raj Shekhar, Children's National Health System (United States)

Jeffrey H. Siewerdsen, Johns Hopkins Univ. (United States)

Stefanie Speidel, Karlsruher Institut für Technologie (Germany)

Andrew D. Wiles, Northern Digital Inc. (Canada)

Robert Webster, Vanderbilt Univ. (United States)

Ivo Wolf, Hochschule Mannheim (Germany)

Ziv R. Yaniv, National Library of Medicine (United States)

Amber Simpson is a computer scientist in the Department of Surgery at Memorial Sloan Kettering (MSK). She specializes in medical image processing and computer-aided surgery, focused on the development of novel computational strategies for cancer treatment. She was recruited to join the MSK faculty as the first PhD in the Department of Surgery. She is a current recipient of an American Association of Cancer Research Career Development Award. She received her PhD from Queen's University in Kingston, Ontario, Canada.

Michael I. Miga received his $\mathrm{PhD}$ in biomedical engineering from Dartmouth College in 1998. He joined Vanderbilt University in spring of 2001 and is the Harvie Branscomb professor at Vanderbilt. He is a professor of biomedical engineering, radiology, and neurological surgery. He is an AIMBE Fellow, director of the Biomedical Modeling Laboratory, and cofounder of the Vanderbilt Institute for Surgery and Engineering. His research interests are in computational modeling and inverse problems for therapeutic applications and imaging. 